\title{
Efeito do tratamento oncológico do câncer de cabeça e pescoço no sistema auditivo
}

\author{
Oncological treatment effect of head and neck cancer on auditory system \\ Efecto del tratamiento oncológico del cancer de cabeza y cuello sobre el sistema auditivo
}

Recebido: 28/12/2021 | Revisado: 04/01/2022 | Aceito: 13/01/2022 | Publicado: 15/01/2022

Priscila Feliciano de Oliveira

ORCID: https://orcid.org/0000-0002-6443-6167

Universidade Federal de Sergipe, Brasil

E-mail: priscila@academico.ufs.br

Amanda Souza de Jesus

ORCID: https://orcid.org/0000-0003-2243-8858

Universidade Federal de Sergipe, Brasil

E-mail: amandasouzafga@gmail.com

Lucas Araujo Reis

ORCID: https://orcid.org/0000-0003-1386-3975 Universidade Federal de Sergipe, Brasil

E-mail: lucas.araujoreis16@gmail.com

Tamara Figueiredo do Carmo Santos ORCID: https://orcid.org/0000-0003-4824-842X Universidade Federal de Sergipe, Brasil E-mail: taam.figueiredo@hotmail.com

Gregorina Silva Ribeiro Rocha ORCID: https://orcid.org/0000-0002-7906-5717 Fonoaudióloga Clínica, Brasil

E-mail: gregorinarocha@gmail.com

Aline Cabral de Oliveira

ORCID: https://orcid.org/0000-0002-8013-8653 Universidade Federal de Sergipe, Brasil

E-mail: alinecabralphd@gmail.com

\begin{abstract}
Resumo
Introdução: A melhoria na qualidade de vida dos pacientes com câncer de cabeça e pescoço tem sido alvo de estudos, entretanto a perda auditiva é um dos efeitos colaterais do tratamento oncológico e afetam significativamente a qualidade de vida do seu portador, principalmente quando é realizada radioterapia combinada com cisplatina. Objetivo: Analisar os achados audiológicos de participantes com câncer de cabeça e pescoço submetidos ao tratamento radioterápico isolado e concomitante a cisplatina. Metodologia: Foram incluídos participantes com diagnóstico anatomopatológico de neoplasia de câncer de cabeça e pescoço que estivessem no final do tratamento oncológico, com limiar auditivo normal prévio ao tratamento antioneoplásico. Dois grupos foram elencados: radioterapia (RT) e quimioterapia concomitante a radioterapia (QTRT). Os participantes do QTRT fizeram uso da cisplatina, droga do grupo da platina com dose entre 200 e 300mg. Foram realizados os seguintes procedimentos: anamnese, meatoscopia e audiometria tonal convencional. Resultados: Participaram 22 sujeitos com média de idade de 48,48 anos, na maioria homens (68,2\%). Verificou-se que 59,1\% apresentaram perda auditiva sensorioneural. O grupo RT foi composto por 10 participantes, $25 \%$ relatou zumbido e $38,5 \%$ foram diagnosticados com perda auditiva. O QTRT com 12 participantes relatou zumbido (25\%) e 61,5\% desenvolveram perda auditiva. Não foi observada diferença estatisticamente significativa entre os grupos para os limiares auditivos nas frequências sob teste. Conclusão: Os pacientes submetidos ao tratamento antineoplásico cursaram com efeitos colaterais significativos relacionados ao órgão da audição, sendo que para o tratamento com quimioterapia e radioterapia concomitante foi significativa a presença de perda auditiva.
\end{abstract}

Palavras-chave: Neoplasias de cabeça e pescoço; Radioterapia; Quimioterapia; Perda auditiva.

\begin{abstract}
Introduction: The improvement in the quality of life of patients with head and neck cancer has been the subject of studies, however hearing loss is one of the side effects of cancer treatment that significantly affect the quality of life of its patient, especially when radiotherapy is performed combined with cisplatin. Objective: To analyze the audiological findings of participants with head and neck cancer who underwent radiotherapy alone and concomitantly with cisplatin. Methodology: Participants with anatomopathological diagnosis of cancer of the head and neck who were at the end of cancer treatment, with normal hearing threshold prior to anticancer treatment, were included. Two groups were listed: Radiotherapy (RT) and chemotherapy concomitant with radiotherapy (QTRT). The QTRT participants used cisplatin, a drug from the Platinum Group at a dose between 200 and 300mg. Among the procedures performed are: anamnesis, otoscopy and conventional pure tone audiometry. Results: 22 subjects participated, with a mean age of 48.48 years,
\end{abstract}


mostly men (68.2\%). It was found that $59.1 \%$ had sensorineural hearing loss. The RT group consisted of 10 participants, $25 \%$ reported tinnitus and $38.5 \%$ were diagnosed with hearing loss. The QTRT with 12 participants reported tinnitus (25\%) and $61.5 \%$ developed hearing loss. There was no statistically significant difference between groups for hearing thresholds in the frequencies under test. Conclusion: Patients undergoing antineoplastic treatment had significant side effects related to the hearing organ, and for treatment with concomitant chemotherapy and radiotherapy, the presence of hearing loss was significant.

Keywords: Head and neck neoplasms; Radiotherapy; Drug therapy; Haring loss.

\section{Resumen}

Introducción: La mejora en la calidad de vida de los pacientes con cáncer de cabeza y cuello ha sido objeto de estudios, sin embargo la hipoacusia es uno de los efectos secundarios del tratamiento oncológico que afecta significativamente la calidad de vida de su paciente, especialmente cuando se trata de radioterapia. realizado combinado con cisplatino. Objetivo: Analizar los hallazgos audiológicos de participantes con cáncer de cabeza y cuello que recibieron radioterapia sola y concomitantemente con cisplatino. Metodología: Se incluyeron participantes con diagnóstico anatomopatológico de cáncer de cabeza y cuello que se encontraban al final del tratamiento oncológico, con umbral auditivo normal previo al tratamiento antineoplásico. Se enumeraron dos grupos: radioterapia (RT) y quimioterapia concomitante con radioterapia (QTRT). Los participantes del QTRT usaron cisplatino, un medicamento del Grupo Platino en una dosis entre 200 y $300 \mathrm{mg}$. Entre los procedimientos realizados se encuentran: anamnesis, otoscopia y audiometría convencional de tonos puros. Resultados: participaron 22 sujetos, con una edad media de 48,48 años, en su mayoría hombres $(68,2 \%)$. Se encontró que el 59,1\% presentaba hipoacusia neurosensorial. El grupo de RT estaba formado por 10 participantes, el 25\% informó acúfenos y el 38,5\% fueron diagnosticados con pérdida auditiva. El QTRT con 12 participantes informó acúfenos (25\%) y el 61,5\% desarrolló pérdida auditiva. No hubo diferencias estadísticamente significativas entre los grupos para los umbrales de audición en las frecuencias bajo prueba. Conclusión: Los pacientes sometidos a tratamiento antineoplásico presentaron efectos secundarios importantes relacionados con el órgano auditivo, y para el tratamiento con quimioterapia y radioterapia concomitantes, la presencia de hipoacusia fue significativa.

Palabras clave: Neoplasias de cabeza y cuello; Radioterapia; Quimioterapia; Pérdida auditiva.

\section{Introdução}

O câncer de cabeça e pescoço corresponde ao grupo heterogêneo de neoplasias que acometem o sistema aerodigestivo superior (Da Silva et al., 2020). Em 2020, representou cerca de $5 \%$ de todos os casos de câncer no mundo e foi responsável por mais de meio milhão de mortes pelo globo (Sung et al., 2021). No Brasil, os números somados de tumores situados na região de cabeça e pescoço correspondem a 6\% do total de câncer no país (INCA, 2019).

O consumo excessivo de álcool e o tabagismo são os principais fatores responsáveis pelo surgimento dessas neoplasias e o uso associado de tais substâncias aumenta em 30 vezes o risco de desenvolver tumores na região (INCA, 2019). Apesar do declínio do tabagismo em vários países, nos últimos anos, a incidência de infecção por HPV tem contribuído significativamente para o surgimento de cânceres, principalmente em orofaringe (Sturgis \& Ang, 2011).

Os tumores de cabeça e pescoço comumente são insidiosos, descobertos em consultas de atenção primária por sintomas inespecíficos ou pelo surgimento de massas cervicais indolores (Yan et al., 2018). Geralmente são diagnosticados em fases avançadas, o que torna necessário tratamentos mais agressivos e impactantes para os pacientes (Cohen et al., 2018).

Atualmente, o tratamento de tumores avançados se baseia principalmente em associação de terapias cirúrgica e quimiorradioterápica, as quais ocorrem de forma individualizada de acordo com as características do tumor e perfil do paciente (Furness et al., 2010; Nichols et al., 2019). A quimioterapia, a base de cisplatina, e a radioterapia são tratamentos de primeira linha para os cânceres de cabeça e pescoço e a utilização das duas terapias associadas apresentam resultados expressivos quanto ao sucesso do tratamento e melhora da sobrevida dos pacientes (Brown et al., 2019; Dellaringa et al., 2010). Apesar do tratamento quimioterápico com uso da cisplatina ser efetivo no combate a estes tipos de neoplasias, é comum o curso de efeitos colaterais, dentre eles, a ototoxicidade (Tchounwou et al., 2021); ademais, a radioterapia também possui efeitos nocivos para o órgão auditivo (Leoncini et al., 2014). É comum observar alterações audiológicas causadas por ambos procedimentos terapeuticos, porém o efeito sinérgico pode afetar o órgão de Corti, e lesionar as células ciliadas externas, as células do gânglio espiral, a estria vascular e ligamento espiral (Cheraghi et al., 2015; Wong et al., 2006). 
É de extrema importância estudos voltados a saúde auditiva do paciente que realiza tratamento oncológico, uma vez que a presença da perda auditiva pode levar o paciente a desencadear desordens emocionais por apresentar dificuldade para efetivar a comunicação oral. Diante do exposto o objetivo deste estudo é analisar os achados audiológicos dos pacientes com câncer de cabeça e pescoço submetidos ao tratamento radioterápico isolado e concomitante a cisplatina.

\section{Metodologia}

Trata-se de um estudo coorte com corte transversal de inquérito, analítico e observacional (Volpato,2007), sendo que todos os preceitos éticos foram seguidos. A pesquisa foi aprovada pelo Comitê de Ética em Pesquisa da Universidade Federal de Sergipe, sob o número 92772318.8.0000.5546 com parecer 2.801.792 e todos os participantes assinaram o Termo de Consentimento Livre e Esclarecido, sendo que foi garantido os direitos de desistência de participação, sigilo e não identificação.

A amostra foi composta por 22 sujeitos adultos de ambos os gêneros, sendo esta de conveniência e foram divididos em dois Grupos: grupo Quimioterapia + Radioterapia (QTRT) e grupo Radioterapia (RT).

Fizeram parte dos critérios de inclusão: diagnóstico anatomopatológico de neoplasia de câncer de cabeça e pescoço, especificamente de laringe e que estivessem no final do tratamento antioneoplásico e que apresentassem limiares auditivos dentro da normalidade anterior ao início do tratamento oncológico. A droga elencada para o estudo foi a cisplatina, droga do grupo da platina com dose entre 200 e 300mg e o procedimento radioterápico preconizado foi o 3D.

Os critérios de exclusão elencados foram: idade superior a 60 anos (para evitar a perda auditiva pela idade, ou seja, presbiacusia), exposição ao ruído/trauma acústico; histórico de cirurgias otológicas e afecções otológicas anteriores a neoplasia; alterações metabólicas (diabetes mellitus, hipertensão arterial); realização de tratamento antineoplásico prévio a presente pesquisa e obstrução de meato acústico externo.

Os participantes foram atendidos em um Hospital Público de Sergipe no setor de oncologia, sob livre demanda, conforme encaminhamento realizado pela equipe multidisciplinar do hospital e os procedimentos aplicados em ambos os grupos foram: anamnese, meatoscopia, audiometria tonal e logoaudiometria.

A anamnese tinha como objetivo a obtenção de dados referentes à identificação do paciente, história pregressa da doença (tempo de descoberta da patologia, sintomas, tipo de neoplasia, tempo de início do tratamento, tempo de utilização da droga e/ou das sessões de radioterapia, dentre outros) e saúde auditiva (presença ou ausência de queixa auditiva e a história pregressa otológica). A anamnese foi aplicada individualmente em uma sala silenciosa. O pesquisador lia em voz alta o questionário e o paciente escolhia a resposta que se enquadrasse no perfil dele. Durante o procedimento, evitou-se a influência do pesquisador nas respostas, sendo que não houve tempo pré-estabelecido para a resposta das perguntas a fim de não induzir o participante. A aplicação do instrumento completou-se em aproximadamente 15 minutos.

A meatoscopia foi realizada para inspeção do meato acústico externo. Quando detectado obstrução, o paciente era encaminhado ao otorrinolaringologista para avaliação e conduta. O equipamento utilizado foi o otoscópio Pocket Junior (WelchAllyn, EUA, modelo 22840).

Para a avaliação auditiva foram realizados os procedimentos de audiometria tonal convencional e logoaudiometria em cabina acusticamente tratada, sendo que foi utilizado um audiômetro de um canal e meio (Interacoustics, Dinamarca, UK, modelo AD229b), com uso de fone TDH 39. Os limiares auditivos foram pesquisados nas frequências interoitavas de $250 \mathrm{~Hz}$ a $8 \mathrm{kHz}$, por via aérea, e de $500 \mathrm{~Hz}$ a $4 \mathrm{kHz}$, por via óssea. O critério de normalidade adotado foi o de Silman e Silverman (1997), que considera os limiares auditivos normais quando os valores estiverem menores ou iguais a 25dBNA. Na logoaudiometria foi realizado apenas o limiar de recepção de fala (LRF) para confirmação dos achados tonais. Um dos critérios de inclusão era audiometria com limiares audiológicos dentro da normalidade, portanto a equipe multidisciplinar encaminhou os portadores de câncer de 
laringe, antes do tratamento oncológico (quimioterapia/radioterapia) para realização da avaliação auditiva a fim da obter o diagnóstico Audiológico.

Foi realizada análise de forma descritiva e inferencial e utilizado o programa Statistical Package for Social Sciences versão 20 (SPSS, Chicago, USA) e a mesma. A descrição das variáveis qualitativas nominais foi realizada por meio de frequência relativa e absoluta. A descrição das variáveis quantitativas foi realizada por meio de medidas de variabilidade (desvio-padrão) e tendência central (média e mediana). Para análise inferencial dos resultados primeiramente foi testada a normalidade da amostra por meio do teste de Smirnov Komorog. Os dados classificados como paramétricos, foram expressos em valores de média \pm desvio padrão da média. Para comparação entre dois Grupos, sob influência de um único fator de variação, foi realizado o teste $T$ de Student e Qui quadrado de Pearson Os resultados classificados como não paramétricos, na comparação entre dois Grupos, sob influência de um único fator de variação, foi realizado o teste de Mann Whitney. A variável dependente (perda auditiva) e as independentes (tipo de tratamento e queixas auditivas). O nível crítico fixado é de $5 \%(\mathrm{p} \leq 0,05)$ para se admitir uma diferença de médias como estatisticamente significante, sendo que $\mathrm{p} \leq 0,10$ foi considerado como tendência a ser significativo.

\section{Resultados}

Participaram da pesquisa 22 sujeitos com câncer de cabeça e pescoço com média de idade de $48,48( \pm 15,15)$ anos, sendo a maioria do gênero masculino $(68,2 \%)$.

O QTRT, composto por 10 sujeitos, apresentou média de idade de $50,70( \pm 12,67)$ anos, sendo a maioria do gênero masculino (70\%). O RT contou com presença de 12 sujeitos, com média de idade de 46,62 $( \pm 17,07)$ anos, sendo que na sua maioria por homens $(66,7 \%)$.

$\mathrm{Na}$ análise da normalidade dos dados verificou-se que os limiares auditivos em todas as frequências não apresentaram diferença estatisticamente significativa com $\mathrm{p}>0,05$, portanto os dados foram considerados dados normais (Teste de Smirnov Komorog). Vale ressaltar que as orelhas direita e esquerda foram agrupadas uma vez que não foi observada na análise estatística diferença estatisticamente significativa com $\mathrm{p}>0,05$ no Teste $\mathrm{T}$ de Student.

Verificou-se que $40,9 \%$ da população estudada (ambos os grupos) apresentaram limiares auditivos dentro os padrões de normalidade, entretanto 59,1\% apresentaram perda auditiva. Os limiares auditivos nas frequências sob estudo podem ser observados na Figura 1.

Figura 1. Limiares auditivos nas frequências sob teste com e sem perda auditiva ( $\mathrm{n}=18$ orelhas com limiares auditivos normais, $\mathrm{n}=26$ orelhas com perda auditiva).

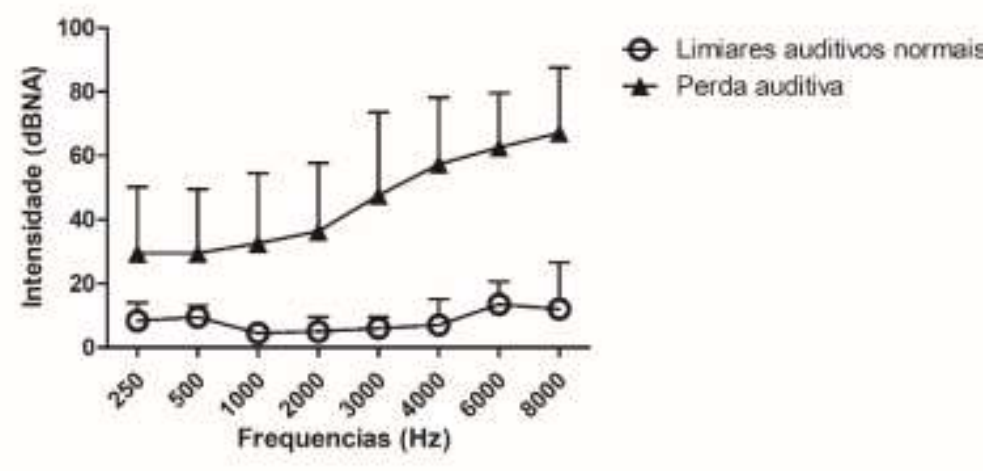

Abreviações: dBNA: decibel nível de audição, Hz : hertz. Fonte: Dados da pesquisa.

Na distribuição dos dados referente aos grupos de estudo, verificou-se que 77,8\% do RT apresentaram limiares auditivos dentro da normalidade, sendo que apenas $22,2 \%$ das orelhas do QTRT apresentaram achados compatíveis com a normalidade. 
Entretanto a Figura 2 descreve que houve diferença significativa para a frequência de $8 \mathrm{Khz}$, sendo que o limiar auditivo do RT foi maior que do QTRT, embora ambos limiares tenham sido classificados como dentro da normalidade.

Figura 2. Efeito do tratamento oncológico para os limiares auditivos normais ( $\mathrm{n}=4$ orelhas do QTRT, $\mathrm{n}=14$ orelhas do RT).

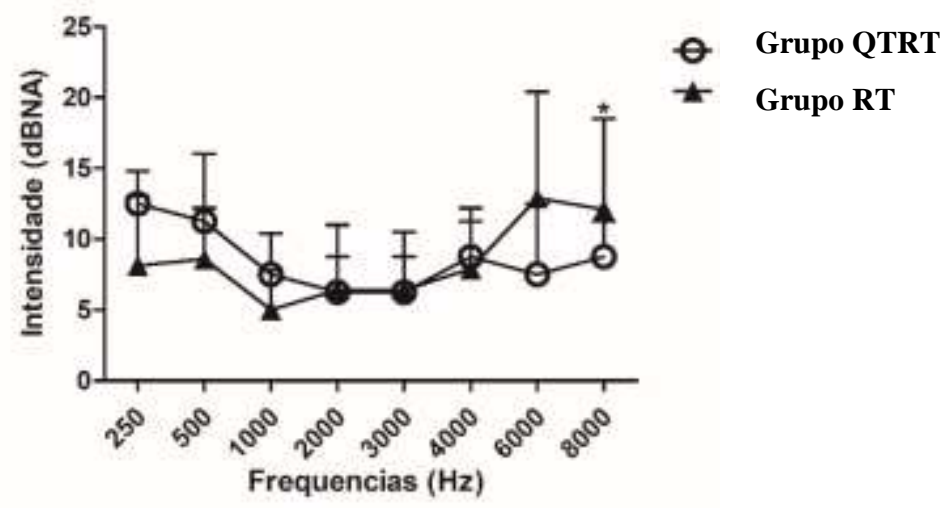

Análise estatística Teste T. Valores expressos com média $\left({ }^{*}\right) p<0,05$ quando comparado ao QTRT. dBNA: decibel nível de audição, QTRT: Grupo quimioterapia + radioterapia, RT: Grupo radioterapia. Fonte Dados da pesquisa.

Ainda relacionado à distribuição dos grupos verificou-se que 61,5\% do QTRT e 38,5\% do RT apresentaram perda auditiva, porém não foi observada diferença estatisticamente significativa entre os grupos para as frequências sob teste (Figura $3)$.

Figura 3. Efeito do tratamento oncológico para os limiares auditivos alterados ( $\mathrm{n}=16$ orelhas do QTRT, $\mathrm{n}=10$ orelhas do RT).

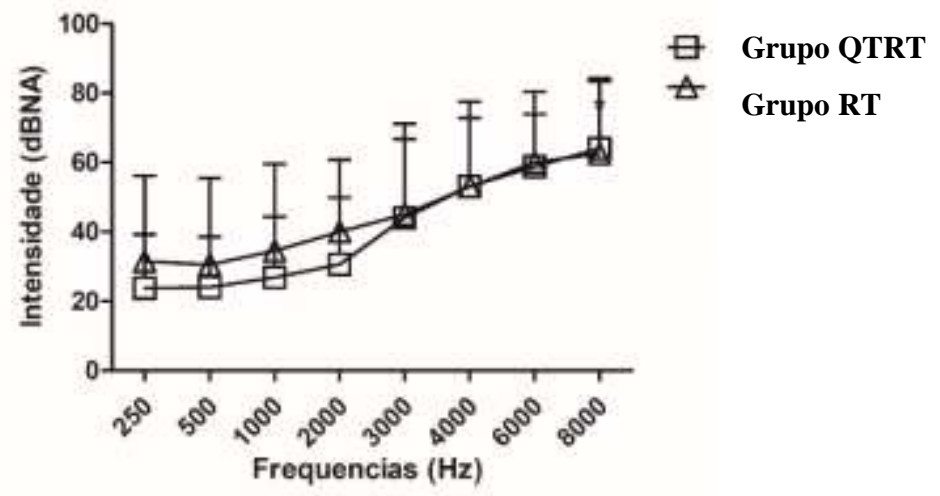

Análise estatística Teste T, valores expressos com. dBNA: decibel nível de audição, QTRT: Grupo quimioterapia + radioterapia, RT: Grupo radioterapia. Fonte: Dados da pesquisa.

Com relação aos sintomas auditivos e dados referente ao câncer nos grupos de estudo tem-se (Tabela 1):

Tabela 1. Dados referentes ao pré e pós-tratamento oncológico dos sujeitos que realizaram quimioterapia e radioterapia concomitantes (QTRT) e radioterapia exclusiva (RT) (n=22).

\begin{tabular}{cccc}
\hline Variável & $\begin{array}{c}\text { QTRT } \\
\mathrm{n}(\%)\end{array}$ & $\begin{array}{c}\text { RT } \\
\mathrm{n}(\%)\end{array}$ & p valor \\
\hline Cirurgia antes do tratamento oncológico & $16(80)$ & $16(66,7)$ & $\mathbf{0 , 0 0 7}^{\text {* }}$ \\
\hline Antecedentes familiares & $8(40)$ & $12(50)$ & 0,371 \\
\hline Alteração na audição pós tratamento & $14(70)$ & $8(33,3)$ & $\mathbf{0 , 0 7 4}^{\#}$ \\
\hline
\end{tabular}

$\mathrm{P}$ valor: * considerado significativo com $\mathrm{p}<0,05 \mathrm{e}^{\#}$ considerado tendência a ser significativo com p $<0,08$ para o Teste Qui Quadrado de Pearson. Fonte: Dados da pesquisa. 
$\mathrm{Na}$ análise dos relatos referente à presença de zumbido, pós tratamento oncológico, observou-se que $25 \%$ de ambos os grupos apresentaram esta queixa, porém o QTRT apresentou uma média de $01( \pm 24,49)$ dia pós tratamento para surgimento do zumbido. Em contrapartida, o RT levou um tempo maior para o paciente sentir a presença de zumbido, sendo em média 36,20 $( \pm 110,73)$ dias. A análise estatística constatou efeito positivo do tempo de sensação do zumbido no QTRT com $p=0,003$ no teste $\mathrm{T}$ de Student.

\section{Discussão}

Na presente pesquisa houve predominância de pacientes do gênero masculino, outrossim, são as estimativas do câncer no mundo, uma vez que a incidência global em 2020 foi $19 \%$ maior para os homens quando comparado às mulheres (Sung et al., 2021). Ademais o cenário nacional estimou 19480 novos casos de câncer de cabeça e pescoço em homens e 17140 em mulheres. Estudos afirmam que os cânceres de cabeça e pescoço são mais comuns no sexo masculino, exceto para o câncer de tireoide, uma vez que a estimativa registra que esse tipo de neoplasia acomete a população feminina 6 vezes mais que a população masculina (INCA, 2019 ).

A média geral de idade, no presente estudo, foi de 48,48 anos, sendo que pacientes submetidos exclusivamente à radioterapia, a média da idade foi ligeiramente menor quando comparado ao QTRT com média de 50,70 anos, compatível com estudos epidemiológicos de câncer de cabeça e pescoço no Brasil. Estudos demonstram idade semelhante quanto ao acometimento do câncer de cabeça e pescoço ao da presente pesquisa (Alvarenga et al., 2008; Faria et al., 2020; Kowalski et al., 2020).

No que diz respeito aos achados audiológicos, 59,1\% apresentaram perda auditiva em algum grau. O estudo de Cheraghi et al. (2015) também evidenciou presença de perda auditiva em 51\% dos sujeitos com tumores de cabeça e pescoço submetidos a regimes de tratamento com radioterapia exclusiva e quimioterapia com cisplatina associada a radioterapia. Em contrapartida, Bhandare et al. (2007), relatou perda auditiva em apenas $15 \%$ da população estudada. A literatura aponta percentuais variados de acometimento auditivo, sendo que a prevalência de ototoxicidade no câncer de cabeça e pescoço varia de $4 \%$ a $90 \%$, dependendo de fatores como agentes concomitantes, doses acumuladas, idade e técnicas de administração (Landier, 2016). Além disso, fatores individuais relacionados a polimorfismo genético também exercem influência na variabilidade do acometimento auditivo (Khrunin et al., 2010).

Observa-se ainda, que o acometimento auditivo também depende da localização do tumor e da dose de radiação necessária para o tratamento. Tumores de seio nasal e parótida, por exemplo, muitas vezes necessitam altas doses de radioterapia, uma vez que estão mais próximos do sistema auditivo e devido à complexidade anatômica da localização de algumas dessas neoplasias, a irradiação no órgão auditivo pode ocorrer com maior intensidade gerando níveis diferentes de lesão auditiva (Mujica-Mota et al., 2013).

Os resultados obtidos na presente pesquisa evidenciaram que 61,5\% dos pacientes tratados com quimiorradioterapia e $38,5 \%$ com radioterapia cursaram com perda auditiva. A literatura corrobora com os achados, uma vez que estudos elucidam maior acometimento auditivo com a associação de quimioterapia e radioterapia (Bhandare et al., 2007; Theunissen et al., 2014; Wong et al., 2006). A ototoxicidade induzida pelo sinergismo das modalidades terapêuticas pode ser explicada tanto pelas características inerentes da cisplatina, ao passo que, sabidamente, é responsável por danos irreversíveis à audição, quanto pelo efeito prejudicial cumulativo da droga (Frisina et al., 2016; Lopes et al., 2020). É possível também que a radiação que ocorra antes ou concomitante à administração da quimioterapia ocasione hiperemia das estruturas do ouvido, o que sensibiliza a cóclea e favorece maior acometimento auditivo sinérgico. Tal fenômeno é justificado em estudos onde a quimioterapia antecedeu os regimes radioativos e foi observado menor número de sujeitos com perda auditiva (Wong et al., 2006). 
As frequências mais afetadas nos pacientes do presente estudo foram acima de $3 \mathrm{kHz}$, ou seja latas ferquencias. A cisplatina gera destruição de células ciliadas externas devido a formação de espécies reativas de oxigênio que atingem a região do ouvido interno e gera perda neurossensorial bilateral e irreversível. Devido a conformidade anatômica da estrutura coclear, os danos ocorrem primeiramente na base da cóclea, região responsável pelo processamento auditivo das altas frequências, o que explica o padrão de acometimento do presente estudo (Landier, 2016). Enquanto que a radiação pode atingir qualquer estrutura do ouvido e gerar desde lesões como estenoses, ulcerações no ouvido externo, otite média, disfunções da tuba auditiva, até lesões vasculares na estrutura coclear, podendo apresentar perda auditiva temporária ou permanente, de caráter condutivo, misto ou neurossensorial (Cheraghi et al., 2015; Jereczek-Fossa et al., 2003).

Foi possível observar divergência ao comparar os achados do presente estudo com os trabalhos encontrados na literatura, uma vez que os limiares auditivos para os pacientes que apresentaram perda auditiva nesta pesquisa foram semelhantes entre QTRT e RT. Entretanto, Cheraghi et al. (2015) e Wong et al. (2006) encontraram limiares auditivos significativamente piores em pacientes que realizaram tratamento associado da quimioterapia a radioterapia quando comparado ao grupo que realizou apenas radioterapia.

Nos sujeitos com limiares auditivos normais foi observado piores resultados no RT em comparação ao QTRT na frequência de $8 \mathrm{kHz}$. Esse resultado pode ser descrito em decorrência da variabilidade de acometimento auditivo já discutido. Em acordo com essa explicação, Cheraghi et al. (2015) demonstrou que as frequências entre 8 e 12kHz foram mais suscetíveis a variações na dose de radiação. Além disso, outra possível explicação são as flutuações na audição descritas na literatura como característica do tratamento radioterápico (Jereczek-Fossa et al., 2003).

A maioria $(80 \%)$ dos pacientes que receberam QTRT fez cirurgia anteriormente. Em contrapartida, os sujeitos que fizeram cirurgia antes da radioterapia somam $66 \%$. Isso pode estar relacionado ao fato de que a radioterapia exclusiva ou a cirurgia em tumores de cabeça e pescoço são terapias de escolha independentes, principalmente nos estágios iniciais de neoplasias como de orofaringe e apresentam resultados clínicos semelhantes do ponto de vista curativo, apesar de possuírem toxicidades diferentes (Nichols et al., 2019).

Já para tumores avançados, terapias combinadas de cirurgia e radioterapia e/ ou quimioterapia geralmente fazem parte do tratamento (Furness et al., 2010). Nesses tumores, a terapia associada de quimiorradioterapia é amplamente utilizada o que pode explicar a predileção de pacientes pós-cirúrgicos necessitarem desse tipo de tratamento em detrimento da radioterapia isolada. Em um estudo de meta análise, sujeitos tratados com quimiorradioterapia apresentaram risco de morte reduzido em relação aos pacientes tratados com esquema de radiação exclusivo. Esses dados podem explicar a maior prevalência de regime de tratamento associado pós-cirúrgico no presente estudo, visto que essa é esta terapia é mais utilizada para tumores mais complexos (Pignon et al., 2009). Ademais, a literatura confirma estes achados, uma vez que a quimiorradioterapia concomitante ao tratamento cirúrgico apresentou melhores resultados em cânceres de cabeça e pescoço quando comparado a radioterapia póscirúrgica. Observa-se um aumento de $22 \%$ na sobrevida global dos pacientes com tumores inoperáveis quando comparado aos pacientes tratados exclusivamente com radioterapia (Furness et al., 2010).

Na presente pesquisa $25 \%$ dos pacientes se queixaram de zumbido. O zumbido é causado principalmente pela perda auditiva neurossensorial, apesar de que qualquer tipo de alteração auditiva também pode desencadear o sintoma (Jereczek-Fossa et al., 2003). A literatura reporta que pacientes tratados com radioterapia em câncer nasofaríngeo, cerca de 50 \% dos pacientes se queixaram de zumbido durante ou após o tratamento e desses pacientes aproximadamente $30 \%$ permaneceram com zumbido intermitente após 1 ano do tratamento radioterápico e cerca de $31 \%$ dos pacientes que não se queixaram durante ou logo após a radioterapia, apresentaram zumbido após 1 ano (Sham \& Choy, 1992). Ademais, fatores psicossomáticos ligados a aspectos emocionais também podem desencadear o sintoma de zumbido (Gibrin et al., 2019). Ademais muitos estudos trazem apenas 
dados referente a presença e ausência de zumbido e não o tempo de surgimento desta queixa, em dias, como descrito na presente pesquisa.

Um das principais problemáticas a presente pesquisa é o pequeno número de participantes. A explicação para isso é que o serviço de audiologia do hospital é o único disponível em todo o estado e os encaminhamentos da equipe multidisciplinar ainda são insuficientes. Além disso, o perfil de morbidade, comum em pacientes com CCP, dificulta a realização das entrevistas e exames audiológicos. Todavia, a limitação mais importante do estudo diz respeito a utilização de audiometria convencional. A literatura é bastante clara em relação ao acometimento inicial ser nas altas frequências, entretanto a audiometria de alta frequência não está disponível no serviço da presente pesquisa. $\mathrm{O}$ exame que avalia as altas frequências é capaz de identificar alterações dos limiares que não são identificadas na audiometria convencional, o que facilita o diagnóstico e intervenção precoce dos pacientes. Sugerimos estudos com audiometria de altas frequências acerca da temática.

\section{Conclusão}

Os efeitos colaterais da quimioterapia e radioterapia são significativos para o órgão da audição, uma vez que a maioria dos sujeitos 59,1\% cursou com perda auditiva. Dentre os procedimentos terapêuticos a quimiorradioterapia com uso da cisplatina foi mais devastador ao órgão da audição com o curso de perda auditiva, entretanto não houve diferença significativa dos limiares auditivos entre os Grupos. Ademais, o zumbido foi uma queixa presente em pacientes de ambas terapêuticas.

Sugerimos a realização de pesquisas acerca desta temática com número de participantes maior e com outros tipos de drogas antineoplásicas, a fim de estudar a relação do maior número possível de drogas concomitante a radioterapia.

\section{Referências}

Alvarenga, L. D. M., Ruiz, M. T., Pavarino-Bertelli, É. C., Ruback, M. J. C., Maniglia, J. V., \& Goloni-Bertollo, E. M. (2008). Epidemiologic evaluation of head and neck patients in a university hospital of Northwestern São Paulo State. Braz.J Otorhinolaryngol. 74(1), 68-73.

Bhandare, N., Antonelli, P. J., Morris, C. G., Malayapa, R. S., \& Mendenhall, W. M. (2007). Ototoxicity after radiotherapy for head and neck tumors. Int J Rad Oncol Biol Physics, 67(2), 469-479.

Brown, A., Kumar, S., \& Tchounwou, P. B. (2019). Cisplatin-Based Chemotherapy of Human Cancers. J Cancer Sci Therapy, $11(4), 97$.

Cheraghi, S., Nikoofar, P., Fadavi, P., Bakhshandeh, M., Khoie, S., Gharehbagh, E. J., Farahani, S., Mohebbi, A., Vasheghani, M., Zare, M., Nikoofar, A., \& Mahdavi, S. R. M. (2015). Short-term cohort study on sensorineural hearing changes in head and neck radiotherapy. Med Oncol, 32(7),200.

Cohen, N., Fedewa, S., \& Chen, A. Y. (2018). Epidemiology and Demographics of the Head and Neck Cancer Population. Oral Maxillofac Surg Clin North America, 30(4), 381-395.

Da Silva, F. A., Roussenq, S. C., Gonçalves de Souza Tavares, M., Pezzi Franco de Souza, C., Barreto Mozzini, C., Benetti, M., \& Dias, M. (2020). Perfil Epidemiológico dos Pacientes com Câncer de Cabeça e Pescoço em um Centro Oncológico no Sul do Brasil. Rev Brasil Cancerol, 66(1), 302-308.

Dellaringa, A. H. B., de Lima Isaac, M., Arruda, G. V., DellAringa, A. R., \& Esteves, M. C. B. N. (2010). Audiological findings in pacients treated with radiotherapy for head and neck tumors. Braz J Otorhinolaryngol, 76(4), 527-532.

Estimativa 2020 : incidência de câncer no Brasil / Instituto Nacional de Câncer José Alencar Gomes da Silva. - Rio de Janeiro : INCA, 2019. (n.d.).

Faria, S. de O., Nascimento, M. C. do, \& Kulcsar, M. A. V. (2020). Malignant neoplasms of the oral cavity and oropharynx treated in Brazil: what do hospital cancer records reveal? Braz Otorhinolaryngol, S1808-8694(20), 30089

Frisina, R. D., Wheeler, H. E., Fossa, S. D., Kerns, S. L., Fung, C., Sesso, H. D., Monahan, P. O., Feldman, D. R., Hamilton, R., Vaughn, D. J., Beard, C. J., Budnick, A., Johnson, E. M., Ardeshir-Rouhani-fard, S., Einhorn, L. H., Lipshultz, S. E., Dolan, M. E., \& Travis, L. B. (2016). Comprehensive audiometric analysis of hearing impairment and tinnitus after cisplatin-based chemotherapy in survivors of adult-onset cancer. J Clin Oncol, 34(23), 2712-2720.

Furness, S., Glenny, A. M., Worthington, H. V., Pavitt, S., Oliver, R., Clarkson, J. E., Macluskey, M., Chan, K. K., Conway, D. I., \& CSROC Expert Panel, U. (2010). Interventions for the treatment of oral cavity and oropharyngeal cancer: chemotherapy. Cochrane database of systematic reviews (Online : Update Software), 9(9), CD006386.

Gibrin, P. C. D., Ciquinato, D. S. de A., Gonçalves, I. C., Marchiori, V. de M., \& Marchiori, L. L. de M. (2019). Tinnitus and its relationship with anxiety and depression in the elderly: a systematic review. Rev CEFAC, 21(4),e7918.

Jereczek-Fossa, B. A., Zarowski, A., Milani, F., \& Orecchia, R. (2003). Radiotherapy-induced ear toxicity. Cancer Treat Rev, $29(5), 417-430$. 
Research, Society and Development, v. 11, n. 1, e56611125209, 2022

(CC BY 4.0) | ISSN 2525-3409 | DOI: http://dx.doi.org/10.33448/rsd-v11i1.25209

Khrunin, A. V., Moisseev, A., Gorbunova, V., \& Limborska, S. (2010). Genetic polymorphisms and the efficacy and toxicity of cisplatin-based chemotherapy in ovarian cancer patients. Pharmacogen J 10(1), 54-61.

Kowalski, L. P., de Oliveira, M. M., Lopez, R. V. M., E Silva, D. R. M., Ikeda, M. K., \& Curado, M. P. (2020). Survival trends of patients with oral and oropharyngeal cancer treated at a cancer center in são paulo, Brazil. Clinics, 75(4),e1507.

Landier, W. (2016). Ototoxicity and cancer therapy. Cancer, 122(11), 1647-1658.

Leoncini, E., Ricciardi, W., Cadoni, G., Arzani, D., Petrelli, L., Paludetti, G., Brennan, P., Luce, D., Stucker, I., Matsuo, K., Talamini, R., La Vecchia, C., Olshan, A. F., Winn, D. M., Herrero, R., Franceschi, S., Castellsague, X., Muscat, J., Morgenstern, H., ... Boccia, S. (2014). Adult height and head and neck cancer: A pooled analysis within the INHANCE Consortium. Head Neck, 36(10), 1391.

Lopes, N. B., Silva, L. A. F., Samelli, A. G., \& Matas, C. G. (2020). Effects of chemotherapy on the auditory system of children with cancer: a systematic literature review. Rev $C E F A C, 22(2), \mathrm{e} 13919$.

Mujica-Mota, M., Waissbluth, S., \& Daniel, S. J. (2013). Characteristics of radiation-induced sensorineural hearing loss in head and neck cancer: A systematic review. Head Neck, 35(11), 1662-1668.

Nichols, A. C., Theurer, J., Prisman, E., Read, N., Berthelet, E., Tran, E., Fung, K., de Almeida, J. R., Bayley, A., Goldstein, D. P., Hier, M., Sultanem, K., Richardson, K., Mlynarek, A., Krishnan, S., Le, H., Yoo, J., MacNeil, S. D., Winquist, E., ... Palma, D. A. (2019). Radiotherapy versus transoral robotic surgery and neck dissection for oropharyngeal squamous cell carcinoma (ORATOR): an open-label, phase 2, randomised trial. Lancet Oncol, 20(10), 1349-1359.

Pignon, J. P., Maître, A. le, Maillard, E., \& Bourhis, J. (2009). Meta-analysis of chemotherapy in head and neck cancer (MACH-NC): An update on 93 randomised trials and 17,346 patients. Radiot Oncol, 92(1), 4-14.

Volpato, G.L. (2007). Ciência da filosofia à publicação. São Paulo: Cultura Academica.

Sham, J. S. T., \& Choy, D. T. K. (1992). Early Changes Of Auditory Brain Stem Evoked Response After Radiotherapy For Nasopharyngeal Carcinoma-A Prospective Study. J Laryngol Otol, 106(10), 887-892.

Sturgis, E. M., \& Ang, K. K. (2011). The epidemic of HVP-associated oropharyngeal cancer is here: Is it time to change our treatment paradigms? J Nat Comp Cancer Net, 9(6), 665-673.

Sung, H., Ferlay, J., Siegel, R. L., Laversanne, M., Soerjomataram, I., Jemal, A., \& Bray, F. (2021). Global Cancer Statistics 2020: GLOBOCAN Estimates of Incidence and Mortality Worldwide for 36 Cancers in 185 Countries. CA: A Cancer J Clinic, 71(3), 209-249.

Tchounwou, P. B., Dasari, S., Noubissi, F. K., Ray, P., \& Kumar, S. (2021). Advances in our understanding of the molecular mechanisms of action of cisplatin in cancer therapy. J Exp Pharmacol, 13, 303-328.

Theunissen, E. A. R., Zuur, C. L., Bosma, S. C. J., Lopez-Yurda, M., Hauptmann, M., Van Der Baan, S., De Boer, J. P., Van Der Molen, L., Rasch, C. R. N., Dreschler, W. A., \& Balm, A. J. M. (2014). Long-term hearing loss after chemoradiation in patients with head and neck cancer. Laryngoscope, 124(12), 27202725 .

Wong, K. L., Song, T. T., Wee, J., Fook-Chong, S. M. C., \& De, Y. W. (2006). Sensorineural hearing loss after radiotherapy and chemoradiotherapy: A single, blinded, randomized study. J Clin Oncol, 24(12), 1904-1909. 6

Yan, K., Agrawal, N., \& Gooi, Z. (2018). Head and Neck Masses. Med Clin North America, 102(6), 1013-1025. 